AB0308 COGNITIVE IMPAIRMENT IN SYSTEMIC LUPUS ERYTHEMATOSUS AND ANTIPHOSPHOLIPID SYNDROME PATIENTS

A. Borisova ${ }^{1}$, T. Lisitsyna ${ }^{2}$, D. Veltishchev ${ }^{1}$, T. Reshetnyak ${ }^{2}$, O. Seravina ${ }^{1}$, O. Kovalevskaya ${ }^{1}$, F. Cheldieva ${ }^{2}$, E. Nasonov ${ }^{3} .{ }^{1}$ Moscow Research Institute of Psychiatry, Serbsky NMRC PN MoH, Department of mental Disorders in Somatic Diseases, Moscow, Russian Federation; ${ }^{2}$ V.A. Nasonova Research Institute of Rheumatology, Vascular Rheumatology, Moscow, Russian Federation; ${ }^{3}$ V.A. Nasonova Research Institute of Rheumatology, Scientific Director of the Institute, Moscow, Russian Federation

Background: Cognitive impairment $(\mathrm{Cl})$ in systemic lupus erythematosus (SLE) and antiphospholipid syndrome (APS) patients have been poorly described and recognized.

Objectives: to describe the rates and spectrum of $\mathrm{Cl}$ in primary (PAPS) and secondary to systemic lupus erythematosus (SLE) (SAPS) APS patients Methods: 113 patients (70 with APS (37 - PAPS, 33 - SAPS) and 43 - SLE without APS), $89(78,8 \%)$ - women, were consecutively enrolled in the study. The mean $(M \pm S D)$ age was $37,9 \pm 11,9$ years. SLE activity was measured by SLEDAI scale. Mental disorders (MD) were diagnosed by psychiatrist in accordance with $\mathrm{ICl}-10$ in semi-structured interview. $\mathrm{Cl}$ were diagnosed with psychology and neuropsychology methods

Results: $\mathrm{Cl}$ of varying severity were found in $105(92,9 \%)$ patients: 62,9\% - mild, $23,8 \%$ - moderate and $13,3 \%$ - severe. Severe and moderate $\mathrm{Cl}$ were more associated with APS $(48,6 \%$ in PAPS and $39,5 \%$ in SAPS vs $18,6 \%$ in SLE, $p=0,004$ and $p=0,04$, accordingly). $\mathrm{Cl}$ were predominantly organic origin in all patients, but vascular dementia was detected only among patients with APS $(10,8 \%$ of PAPS and $3,03 \%$ of SAPS patients). There was no association of $\mathrm{Cl}$ with clinical manifestations and SLE activity. In patients with PAPS CI was associated with stroke, livedo reticularis and lupus anticoagulant positivity. In 84 patients $(74,3 \%) \mathrm{Cl}$ were also specifically bounded to MD. Current MD were detected in $100(88,5 \%)$ patients: schizotypal disorder was found in $10(8,85 \%)$ patients and was associated with PAPS (13,5\% vs $9,09 \%$ in SAPS and $4,65 \%$ in SLE); anxiety-depressive spectrum disorders (ADDs) - in $95(84,1 \%)$ (chronic and recurrent depression prevailed $37(32,7 \%)$ and $42(37,2 \%)$ resp.); the structure of MDs in accordance with $\mathrm{ICl}-10$ differed slightly between groups, but no statistically significant differences were obtained.

Conclusion: cognitive impairment, mainly of an organic type, are characteristic of most patients with SLE and APS. The significant associations of cognitive impairment with clinical manifestations and activity of SLE were not identified, but patients with cognitive impairments were more likely to have anxiety and depressive disorders, strokes, livedo reticularis and lupus anticoagulant positivity Disclosure of Interests: None declared

DOI: 10.1136/annrheumdis-2021-eular. 1402

\section{AB0309 \\ SAFETY OF 23-VALENT POLYSACCHARIDE PNEUMOCOCCAL VACCINE IN PATIENTS WITH SYSTEMIC LUPUS ERYTHEMATOSUS}

G. Tarasova ${ }^{1}$, B. Belov ${ }^{1}$, M. Cherkasova ${ }^{2}$, E. Aseeva ${ }^{3}$, T. Reshetnyak ${ }^{4}$, N. Kosheleva ${ }^{4}$, T. Popkova ${ }^{5} .{ }^{1}$ V.A. Nasonova Research Institute of Rheumatology, Laboratory for the Study of Comorbid Infections and Monitoring the Safety of Drug Therapy, Moscow, Russian Federation; ${ }^{2}$ V.A. Nasonova Research Institute of Rheumatology, Laboratory of Immunology and Molecular Biology of Rheumatic Diseases, Moscow, Russian Federation; ${ }^{3}$ V.A. Nasonova Research Institute of Rheumatology, Intensive Therapy Laboratory, Moscow, Russian Federation; ${ }^{4}$ V.A. Nasonova Research Institute of Rheumatology, Vascular Rheumatology Laboratory, Moscow, Russian Federation; ${ }^{5}$ V.A. Nasonova Research Institute of Rheumatology, Systemic Rheumatic Diseases Laboratory, Moscow, Russian Federation

Background: Vaccination of patients with autoimmune diseases with pneumococcal vaccines is necessary to prevent severe respiratory infections in this group of patients. The main issue of immunization of patients with systemic lupus erythematosus (SLE) remains the issue of safety.

Objectives: The aim of the study was to study the safety of the 23-valent polysaccharide pneumococcal vaccine (PPV-23) in patients with SLE.

Methods: The study included 73 patients with a reliable diagnosis of SLE, of which women -64, men - 9, aged 19 - 68 years. 69 patients received glucocorticoids (GC) $5-30 \mathrm{mg} /$ day, 55 - hydroxychloroquine (GCH), 37-cytostatics (CS), 27 - biologics (14 - rituximab (RTM), 11 - belimumab (BLM), 2-BLM and RTM). 1 dose $(0.5 \mathrm{ml})$ of PPV23 was administered subcutaneously. 60 patients were examined within 1 year, 13 - within 2-3 months.

Results: Vaccination tolerance was assessed in 73 patients: in $33(45.2 \%)$ vaccine reactions were absent, in $36(49.3 \%)$ - local reactions of mild and moderate severity were noted (pain, swelling, skin hyperemia at the injection site of the vaccine), lasting from 2 to 7 days, in $1(1.4 \%)$ - general weakness within 1 month, in $2(2.7 \%)$ - mild diarrhea within 1 day. Vaccinal reactions were typical and completely reversible, did not require additional appointments. One patient (1.4\%) developed a hyperergic reaction of the Artyus phenomenon type, which was arrested within 7 days by the use of antihistamines and topical GCs. None of the 60 patients, whose follow-up period was 1 year, had no exacerbations of the disease directly related to vaccination (i.e., in the next 2-3 months). Vaccination was carried out both at a low degree of activity $(n=33(55 \%))$ and remission $(n=$ $6(10 \%))$, and at an average $(n=12(20 \%))$ and high $(n=9(15 \%)))$ the degree of SLE activity. The dynamics of the SLE activity index SLEDAI-2k (Me) during the year was as follows: initially - $4(2 ; 6)$, after 2-3 months - $2(2 ; 4)$, after 12 months $2(2 ; 4)$. During the year, 7 out of 60 patients had a moderate exacerbation of the disease, which was not related to the vaccination in terms of timing: after 3.5-5 months (3), 12 months (4). An exacerbation occurred in 4 - with a decrease in the HA dose, in 1 - after psychological stress, in 1 - against the background of persistently high immunological activity and insufficient therapy, in 1 - without an increase in immunological activity. In 4 out of 7 , exacerbation was manifested by skin rashes and articular syndrome, in 1 - by the development of panniculitis, in 2 - by leukopenia. All these symptoms were noted earlier in the period of exacerbation. In all, the exacerbation was quickly stopped by a moderate increase in the HA dose. In 60 patients, the dynamics of immunological markers of SLE was analyzed during the year after vaccination. There was no evidence of a significan increase in the immunological activity of SLE after vaccination with PPV-23. After vaccination, no new autoimmune phenomena have been identified. In the first 3 months. after vaccination, in isolated cases, there was a transient increase / decrease in SLE markers (a-DNA, ANF, C3, C4) with a subsequent return to the initial values, without symptoms of exacerbation of the disease.

Conclusion: 1. During the year of observation, no exacerbations were observed that were reliably associated with vaccination. 2 . Vaccination with PPV-23 is safe for SLE patients during periods of low and moderate activity. If necessary, vaccination is possible at high activity without the development of adverse events. 3 The multidirectional dynamics of the main markers of SLE observed during the year reflects the instability of immunological parameters characteristic of SLE.

Disclosure of Interests: None declared

DOI: 10.1136/annrheumdis-2021-eular.1440

\section{$\mathrm{AB} 0310$ \\ TOLERABILITY AND SAFETY OF 23-VALENT POLYSACCHARIDE PNEUMOCOCCAL VACCINE IN PATIENTS WITH ANTIPHOSPHOLIPID SYNDROME, PRELIMINARY RESULTS}

G. Tarasova ${ }^{1}$, B. Belov ${ }^{1}$, M. Cherkasova ${ }^{2}$, E. Aseeva ${ }^{3}$, T. Reshetnyak ${ }^{4}$, N. Kosheleva ${ }^{4}$, T. Popkova ${ }^{5} .{ }^{1}$ V.A. Nasonova Research Institute of Rheumatology, Laboratory for the Study of Comorbid Infections and Monitoring the Safety of Drug Therapy, Moscow, Russian Federation; ${ }^{2}$ V.A. Nasonova Research Institute of Rheumatology, Laboratory of Immunology and Molecular Biology of Rheumatic Diseases, Moscow, Russian Federation; ${ }^{3}$ V.A. Nasonova Research Institute of Rheumatology, Intensive Therapy Laboratory, Moscow, Russian Federation; ${ }^{4}$ V.A. Nasonova Research Institute of Rheumatology, Vascular Rheumatology Laboratory, Moscow, Russian Federation; ${ }^{5}$ V.A. Nasonova Research Institute of Rheumatology, Systemic Rheumatic Diseases Laboratory, Moscow, Russian Federation

Background: Antiphospholipid syndrome (APS) is an autoimmune disease often associated with severe, life-threatening vascular complications. The majority of patients (and in case of secondary APS, in 100\% of cases) receive immunosuppressive therapy. Immunization with pneumococcal vaccines in patients with both primary APS (PAPS) and APS+SLE or secondary (SAPS) is necessary to prevent severe respiratory infections in these patients.

Objectives: Purpose of the study - to study the tolerance and safety of 23-valent polysaccharide pneumococcal vaccine (PPV-23) in patients with PAPS and SAPS.

Methods: At this stage, the study included 28 patients with APS, of which 10 with PAPS, 18 with sAPS proceeding against the background of systemic lupus erythematosus (SLE), of which 23 women (82\%), 5 men (18\%). The average age $(\mathrm{Me})$ of patients was $43(35.5 ; 53.0) \mathrm{g}$. 20 patients received glucocorticoids (GC) $5-30 \mathrm{mg} /$ day equivalent to prednisone, 17- hydroxychloroquine, 6- cytostatics (3-cyclophosphamide, 2-azathioprine, 1- mycophenolate mofetil), 8- biologics: 5-rituximab (RTM), 3-belimumab (BLM); 20-received anticoagulants (direct-10 indirect-10).

1 dose $(0.5 \mathrm{ml})$ of PPV-23 was administered subcutaneously. The follow-up time was 1 year in 23 patients and 5-5.5 months in 5-5.5 months. During the visits, standard clinical and laboratory tests were performed, immunological blood test and the level of antibodies to S.pneumoniae

Results: Vaccination was well tolerated in all patients. In $29 \%$ of cases, vaccine reactions of mild severity were observed: in 7 (25\%) - a local reaction (pain in the arm for 1-3 days-at 7, redness up to $2 \mathrm{~cm}$ at the injection site-at 1$)$, in $1(3,6 \%)$, the patient experienced general weakness (moderately pronounced) for 1 month. Vaccinal reactions were completely reversible and did not require additional prescriptions. Post-vaccination complications develop, as a rule, in the first 1-2 